\title{
Lifetime prediction of vehicle components using online monitoring data
}

\author{
Chiharu Kumazaki*1, Watalu Yamamoto ${ }^{2}$, Kazuyuki Suzuki ${ }^{2}$ \\ 1. Hino Motors, Ltd., 3-1-1, Hino-dai, Hino, Tokyo, Japan, 191-8660. \\ 2. The University of Electro-Communications: 1-5-1, Chofugaoka, Chofu, Tokyo, Japan, 182-8585. \\ *contact author’s e-mail address: chi.kumazaki@hino.co.jp
}

\begin{abstract}
:
In order to set an adequate lifetime target for each market, quantitative evaluation of variation of lifetime characteristics is required. In particular, the lifetime of vehicle unit depends heavily on customer's usage (e.g. gross vehicle weight, road gradient, acceleration operation). We thus have developed an online monitoring system that continually collects some information such as usage and environmental conditions. A method has been developed for predicting vehicle component lifetimes using data from an online monitoring system that collects an extensive amount of data during vehicle operation. The linear model used for prediction takes into account variations in usage conditions and models data as covariates. The prediction procedure was generalized to enable it to make predictions using a new data sample. The large amount of information on usage and environmental conditions obtained with the online monitoring system enabled the usage of each sample to be quantified and treated as a stratification factor. A stratified analysis produced fairly accurate results, meaning that using online monitoring data should be useful for lifetime prediction.
\end{abstract}

\section{Keywords}

Cumulative Damage Rule, Linear Regression Model, Principal Component Analysis, Stratified Analysis, Usage Conditions

\section{Introduction}

\subsection{Recent Approach for Reliability Lifetime Design}

The lifetimes of vehicle components greatly depend on the usage conditions. This is particularly true for commercial vehicles as they are often operated under severe conditions. Improvement in lifetime design for each market requires an understanding of the relationship between the lifetime characteristics and usage conditions in the actual market. If it is not done correctly, we will not prevent the unexpected failure and further trouble. However, the conventional market research requires on-the-spot measurement of actual vehicles. Therefore the research has been conducted with both a small number of samples and non-random sampling, and we did not completely understand the market. In the recent situation of globalization, the importance of market research has increased than ever before. We thus have developed an online monitoring system that continually collects vehicle information during operation by in-vehicle communication terminal. This system enables an extensive amount of vehicle driving data, with a larger sample size and a longer observation period, to be easily collected. The data can be used to clarify the actual usage conditions. From this market information, we performed lifetime design suitable for each market.

Utilization of market information from online monitoring has been attracting attention in various fields. In the reliability field, as a case study to utilize such a "Big Data", some applications have been introduced by Suzuki(2014) in the 1st forum in 2014 of the Reliability Engineering Association of Japan. In this forum, important industries in Japan such as construction machine, aircraft, copying machines and automobiles were introduced. So, utilization of the online monitoring data has become important to improve the reliability of our products.

\subsection{Recent Study for Using Online Monitoring}

The purpose of this study is to feed back to the appropriate lifetime design using these online monitoring data. To systemize the scheme to clarify the usage and modeling the relationship between lifetime and usage, this 
study continues to analyze the data. In the recent study for online monitoring data, Suzuki (2014) generalized a structure for online monitoring data and stated the importance of online monitoring based on the physical and scientific mechanism. According to this paper, to prevent the unexpected troubles, it is necessary that the failure mode is observed. If the failure mode cannot be observed directly, we may be able to predict it from an alternative characteristic value for failure. For example, the major alternative characteristic value for many vehicle components is the load frequency (torque transfer to the driveline, i.e., input torque). If a failure mechanism is revealed, the failure mode can be reliably predicted theoretically. However, in practice, even the measurement of the load frequency is difficult in many cases. This means that various usage and environmental conditions during operation are important pieces of information for describing the characteristics of failure. Use of online monitoring system to collect such information facilitates the collection of a large amount of data.

As an actual analysis case, Meeker and Hong (2014) described several products in which online monitoring data were used for reliability purposes. This study dealt the data of usage conditions as the SOE (System Operating and Environmental) data, and has stated the importance of these data as the information that indicating the sign of failure or deterioration. SOE data is dynamic covariates, and can predict lifetime characteristics in real time based on time-series analysis. By contrast, Lee, et al. (2014) and Pecht and Gu (2009) described the study of PHM (Prognostics and Health Management). PHM is the methods that can be diagnose the sign of failure, and evaluate the health state from covariates directory related to the failure mechanism. They focused on observation of information about the failure, and advanced failure analysis based on the alternative characteristic value of the failure mode in case of various products. On the other hand, since the analysis of SOE data is required to understand the theory of the time-series analysis and the methods of PHM require the measurement of the alternative characteristic values of the failure mode, it is still difficult to apply these studies to the actual development site. Thus, Kumazaki et al. (2012) discussed the utilization of online monitoring based on the analysis of actual data. This study analyzed SOE data without considering as time-series data, and predicted product lifetime using various covariates by applying a linear regression model. Multiple regressions are useful method as the most simple and easy way for utilizing multivariate online monitoring data.

In this study, we have developed a method for predicting vehicle component lifetimes using data from an online monitoring system that collects an extensive amount of data during vehicle operation. In the same way as Kumazaki et al.(2012), the linear regression model used for prediction takes into account variations in usage conditions and models data as covariates, and this model was able to predict the lifetime of the individual component with a high accuracy. However, since its model represents only the average effect of covariates, there is a limit to the linear model to try to predict the overall market. Most of the studies dealt with online monitoring data focused on not so much prediction of the lifetime characteristics for the overall market as prediction accuracy for individual samples. Then, this study, from the viewpoint as the actual developer's side, considered an extension of the linear regression model to be able to predict the overall market. A stratified analysis produced fairly accurate results, meaning that using online monitoring data should be useful for predicting component lifetimes.

\section{Online Monitoring for Commercial Vehicles}

\subsection{Target Component}

The component targeted was the transmission $(T / M)$ and the focus was on gear surface failure due to pitting or spalling. The relationships among failure mode, failure mechanism, and usage conditions for the $T / M$ gears are illustrated in Figure 1 based on the schemes of Suzuki (2014).

The failure mode of gear surface failure is fatigue. Therefore, the failure mechanism of gear failure is specified by the load stress to the drive-train of vehicles. This mechanism is quantified by the cumulative damage rule and the $S-N$ curve. These measures are explained in detail in JSME S 002 (1994). 
Lifetime prediction using monitoring data, Kumazaki et al.

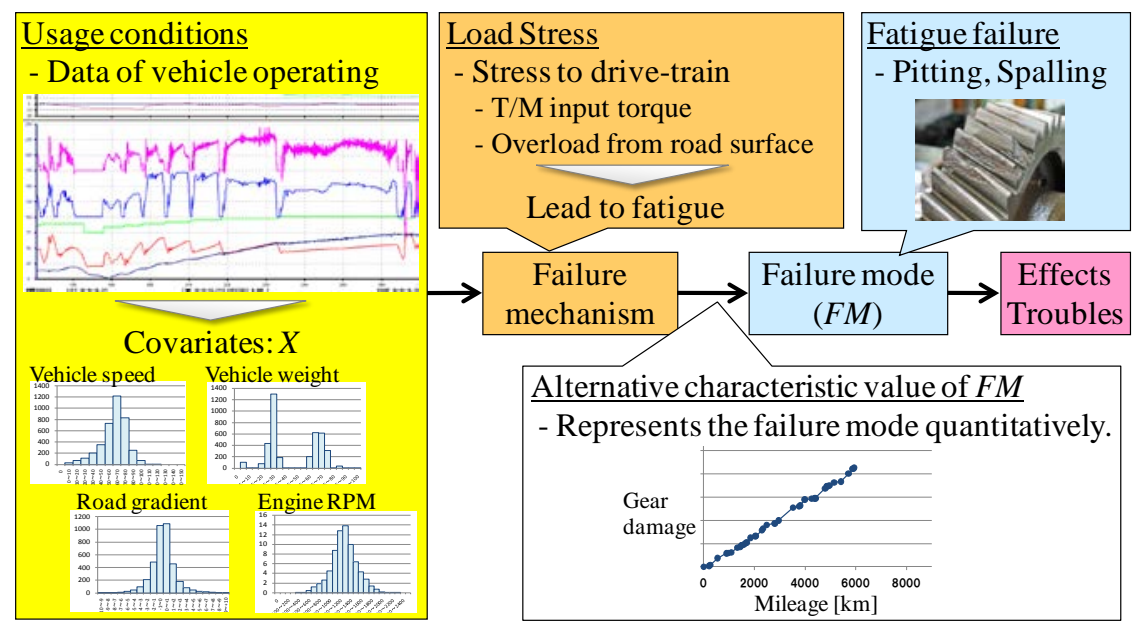

Figure 1: Usage conditions, failure mechanism, and failure mode

\subsection{Online Monitoring Data}

The data of usage conditions called "Covariate" includes the vehicle speed $[\mathrm{km} / \mathrm{h}]$, the gross vehicle weight (GVW) [ton], the road gradient [\%], the accelerator position angle [\%], the engine speed [rpm], the current gear position, the number of upshifts, the number of downshifts, and the number of vehicle starts. These data were obtained as frequency distributions. For each one, we obtained a covariate, either the average value or the standard deviation of the distribution.

The data were collected from 12 vehicles operating in a certain area. The vehicles had the same type of $T / M$, and the gear to be analyzed was the same for each vehicle. As shown in Table 1, each vehicle was operated by one of four participating transport companies (2-4 vehicles per company). The vehicle's data has been monitored for 4 months from January to April 2014.

Table 1: Selected covariates and their meanings

\begin{tabular}{l|ccc|ccc|cc|cccc}
\hline Vehicle No. & 1 & 2 & 3 & 4 & 5 & 6 & 7 & 8 & 9 & 10 & 11 & 12 \\
\hline Vehicle ID & A1 & A2 & A3 & B1 & B2 & B3 & C1 & C2 & D1 & D2 & D3 & D4 \\
\hline Company ID & \multicolumn{3}{|c|}{} & A & \multicolumn{3}{c|}{ B } & \multicolumn{3}{c}{ C } & \multicolumn{4}{c}{ D } \\
\hline
\end{tabular}

\subsection{Definition of Gear Damage and Lifetime Prediction}

We first discuss the prediction of gear lifetime using the relationship between failure mode (fatigue) and failure mechanism (load stress). The load frequency distribution can be illustrated as shown in Figure 2. This figure shows the number of gear rotations $\left\{n_{1}, n_{2}, \ldots, n_{\mathrm{k}}\right\}$ for each torque range $\left\{s_{1}, s_{2}, \ldots, s_{k}\right\}$ (less than $s_{1}$ is the fatigue limit, which is not considered damage).

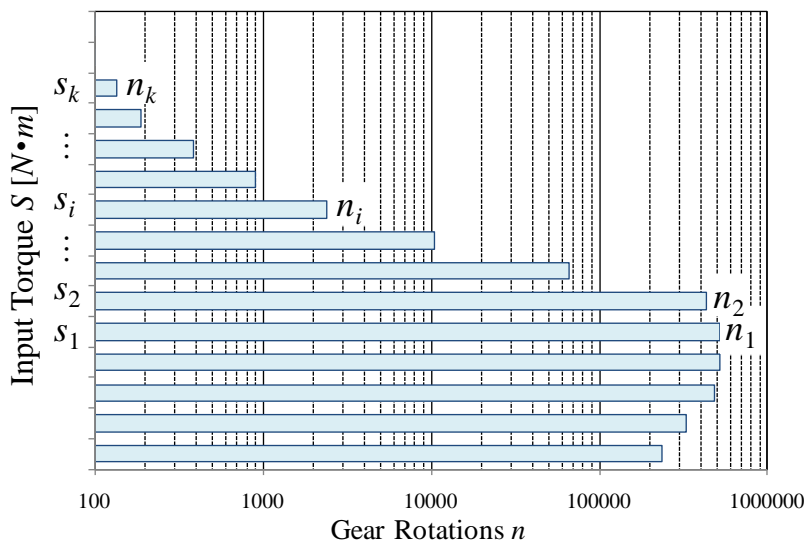

Figure 2: Distribution of load frequency 
When the load frequency was obtained after operating $t[\mathrm{~km}]$, the gear damage is calculated as

$$
y(t)=\sum_{i=1}^{k} n_{i} / N_{i}
$$

with a cumulative damage rule. When the degree of damage reaches 1 , the gear is assumed to have failed. From this relationship, we thus deal this value as an alternative characteristic value that represents the failure mode quantitatively. Predicted lifetime $L$ is calculated using

$$
L=t / y(t)
$$

\subsection{Damage for Sample Data by Cumulative Graph}

The cumulative graph in Figure 3 illustrates the state of progress (accumulation) of the degree of damage. The vertical axis represents cumulative damage $y$ for each measurement point, and the horizontal axis represents cumulative mileage $t[\mathrm{~km}]$. Each line represents one sample path, and contains 15 measurement points. The measurements were made every week for 15 weeks (sample $C 2$ was an exception with only 13 points)

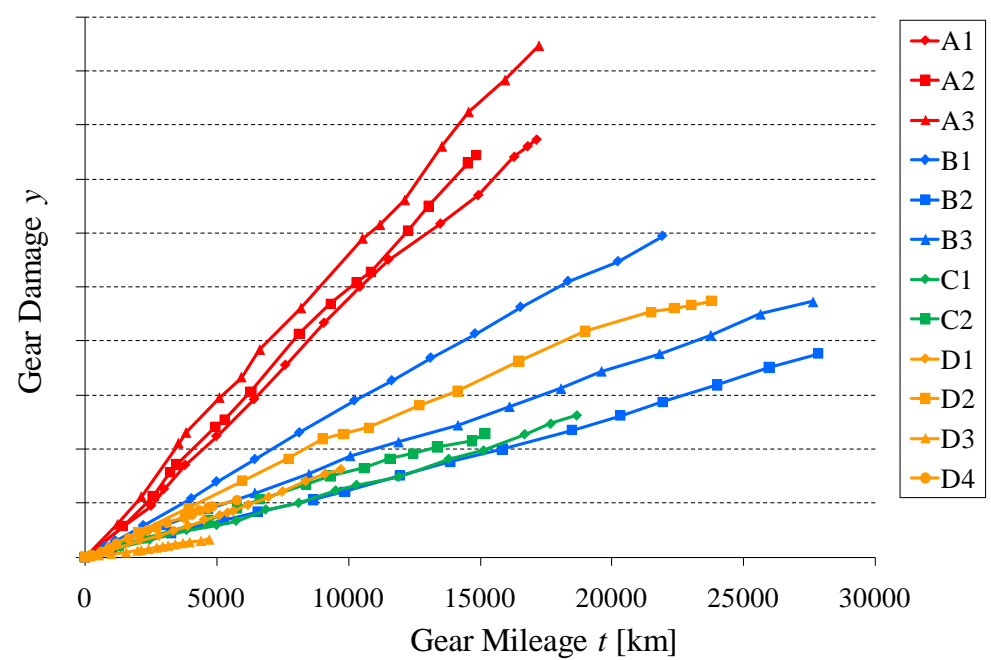

Figure 3: Cumulative degree of gear damage for each measurement point

A change in the long-term trend of a line would indicate a possible large change in the operating conditions, such as a change in the driver or a change in the usage conditions. The lines for our samples remained fairly linear. As mentioned in Section 2.3, when the degree of damage $y$ reaches 1, the gear is assumed to have failed. This point can be easily predicted by extrapolation using linear regression:

$$
y=a \cdot t+\varepsilon .
$$

\section{Lifetime Prediction Using Usage Data}

\subsection{Model of Degree of Damage}

In the previous section, we described prediction of the degree of damage and lifetime from the load frequency as the alternative characteristic value for failure. Here we describe prediction of the degree of damage from the usage data. First, the degree of damage for measurement point $j$ of sample $i$ is given as

$$
\begin{aligned}
& y\left(t_{i j}\right)=y\left(t_{i \cdot j-1}\right)+\alpha_{i j} \times\left(t_{i j}-t_{i \cdot j-1}\right), \\
& i=A 1, A 2, \ldots, D 4, j=1,2, \ldots, n_{i},
\end{aligned}
$$

by adding an increment to the previous point $j-1$. The $\alpha_{i j}$ is the degree of damage per unit mileage at each measurement point. Because the degree of damage depends on the mileage, equation 4 isolates the effect of mileage by introducing $\alpha_{i j}$, which is formulated using a linear regression model: 


$$
\begin{aligned}
& \ln \boldsymbol{\alpha}_{i j}=\mathbf{X}_{i j} \boldsymbol{\beta}_{i}+\boldsymbol{\varepsilon}_{i j}, \\
& \boldsymbol{\varepsilon}_{i j} \sim N\left(\mathbf{0}, \sigma_{e}^{2} \mathbf{I}\right), \mathbf{X}_{i j}=\left[\mathbf{1}, \mathbf{x}_{1 i j}, \ldots, \mathbf{x}_{p i j}\right] \boldsymbol{\beta}_{i}=\left[\beta_{0 i}, \beta_{1 i}, \ldots, \beta_{p i}\right]^{\prime},
\end{aligned}
$$

where $p$ is the number of covariates. In contrast to Figure 3, Figure 4 shows the trend in $\ln \alpha$. Equation 5, which is a linear model, treats these data as objective variables.

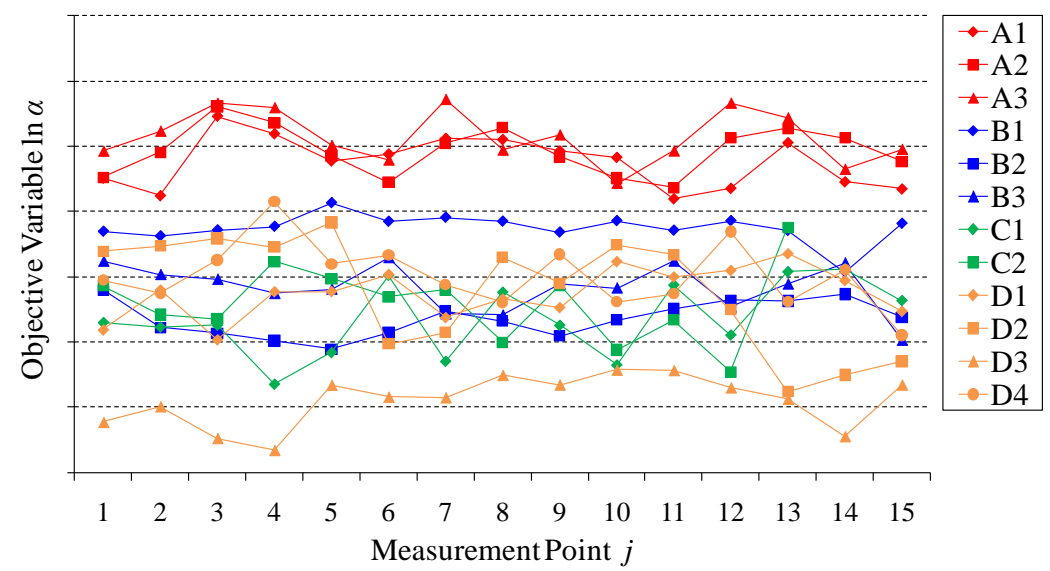

Figure 4: Objective variable $\ln \alpha$

\subsection{Selection of Covariates}

The selection of covariates $x=\left[x_{1}, x_{2}, \ldots, x_{p}\right]$ is important in formulating a linear model. There are several statistical approaches to covariate selection, such as stepwise selection. However, correlation is complicated in the analysis of online monitoring data. Therefore, creating a meaningful model using only these selection methods is difficult. We thus identified five covariates that could be used instead as shown in Table 2.

Table 2: Selected covariates and their meanings

\begin{tabular}{l|l}
\hline Avg. GVW & Average of distribution of gross vehicle weight (GVW) \\
\hline Gradient & Percentage of running time when road gradient is 3\% or more \\
\hline Avg. Speed & Average of distribution of vehicle speed \\
\hline Avg. Accelr Pos. & Average of distribution of accelerator position angle \\
\hline Start Freq. & Number of start times per km \\
\hline
\end{tabular}

\subsubsection{Selection Process}

The selection process was as follows. First, the load applied to the vehicle's driveline is considered to be the "running resistance" input from the tire. Running resistance is divided into "rolling resistance," "grade resistance," and "air resistance," which are respectively proportional to vehicle weight, road gradient, and vehicle speed. For a driver, the direct input device for the driveline is the accelerator pedal. Since engine torque changes with the operation of the pedal, pedal operation contributes to the load on the driveline. Also considered is the higher instantaneous load that occurs when the vehicle is started or the transmission is shifted (this load is called "shock torque").

\subsubsection{Correlation of Covariates}

A scatter plot matrix based on the data for sample $A 1$ is shown in Figure 5. The correlations between the covariates, which are shown in the upper right part of the figure, are reasonably small. Table 3 shows the tolerance and the variance inflation factor (VIF) calculated from the same data. These values are often used as an index of multicollinearity. Since the tolerance values are 0.1 or more and the VIF values are less than 10, there is no multicollinearity between the covariates. 
Total Quality Science Vol.1, No.2

Table3: Multicollinearity (for sample A1)

\begin{tabular}{lcc}
\hline & Tolerance & VIF \\
\hline Avg. GVW & 0.207 & 4.839 \\
Gradient & 0.436 & 2.294 \\
Avg. Speed & 0.184 & 5.436 \\
Avg. Accelr Pos. & 0.306 & 3.267 \\
Start Freq. & 0.421 & 2.376 \\
\hline
\end{tabular}

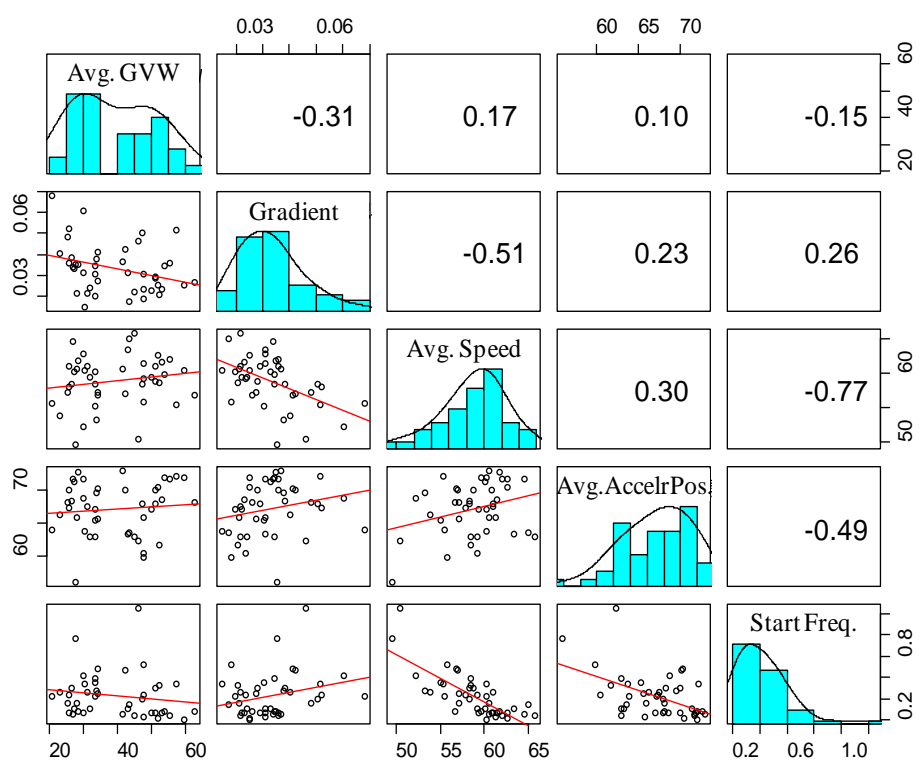

Figure 5: Scatter plots and correlations (sample $i=A 1, n=40$ )

\subsection{Estimation Results for Linear Regression Model}

The results of estimation for sample $A 1$ are shown in Table 4 and Figure 6 . The adjusted R-squared value (0.97) is high enough for practical purposes, and the predicted values were in good agreement with the actual values.

Table 4: Estimation results using equation 4 (for sample $A 1$ )

\begin{tabular}{l|cc}
\hline & Coef. & $p$-value \\
\hline Intercept & -13.985 & $<0.000$ \\
\hdashline Avg. GVW & 0.005 & 0.075 \\
Gradient & -2.001 & 0.081 \\
Avg. Speed & -0.013 & 0.019 \\
Avg. Accelr Pos. & 0.043 & $<0.000$ \\
Start Freq. & 0.326 & 0.002 \\
\hline
\end{tabular}

\begin{tabular}{ll}
\hline Residual Standard Err. & 0.032 \\
\hline Adjusted R-squared & 0.970 \\
\hline
\end{tabular}




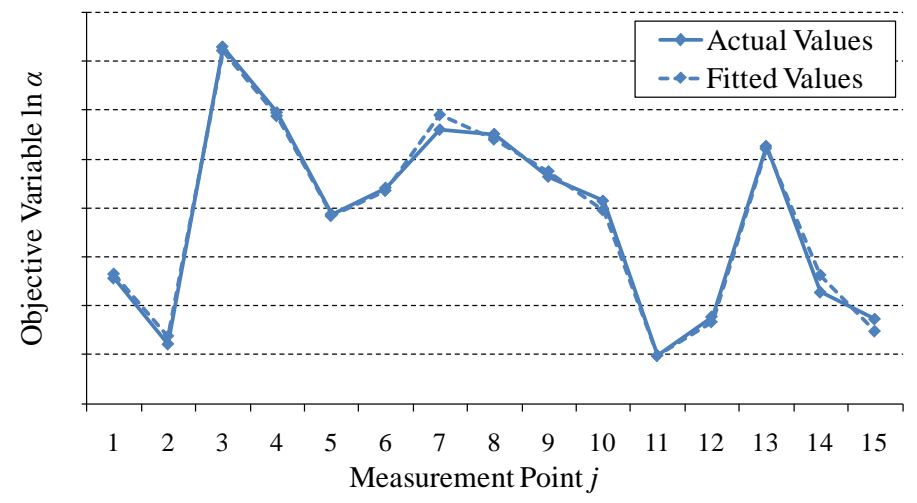

Figure 6: Estimation results for objective variables $\ln \alpha$ (for sample A1)

\subsection{Predicted Lifetime Results}

The relative error

$$
\frac{\left|L_{a}-L_{b}\right|}{L_{a}} \times 100[\%]
$$

is calculated from lifetime $L_{a}$ obtained directly from the load frequency (solid line in Figure 6) and lifetime $L_{b}$ obtained from the linear model (dashed line in Figure 6). We used the relative error as an index of prediction accuracy.

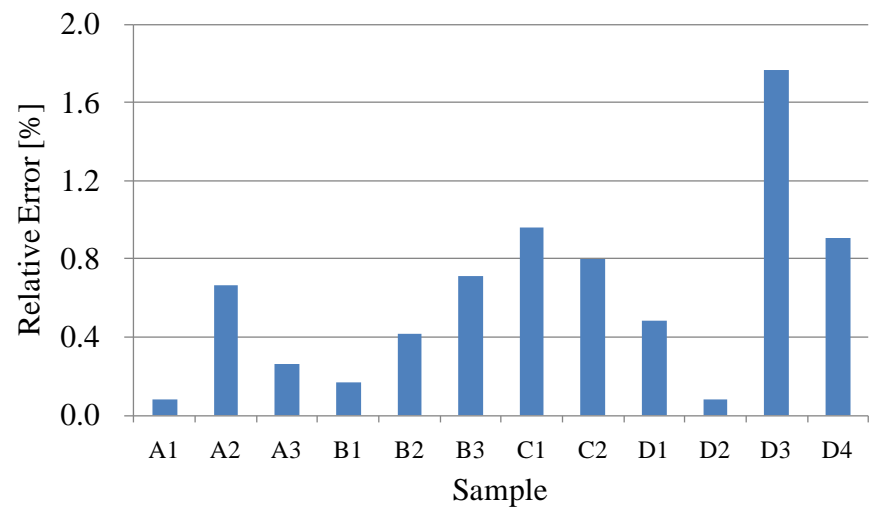

Figure 7: Relative error in predicted lifetime [\%] (Derived from equation 5)

As shown in Figure 7, the relative error was less than 1\% except for sample $D 3$. This means that the lifetime can be predicted from easily collectable usage data with about the same accuracy as with using the load frequency. Furthermore, the simple linear model used can be easily applied to actual product design and development.

\section{Model Extension}

\subsection{Generalization of Prediction Procedure}

The previous section presented the results for one sample. For the model to be practical, it should be able to handle a new sample as well. We thus applied the model to all 12 samples. Table 5 and Figure 8 show the results of estimation using

$$
\ln \boldsymbol{\alpha}_{i j}=\mathbf{X}_{i j} \boldsymbol{\beta}+\boldsymbol{\varepsilon}_{i j}, \quad \boldsymbol{\beta}=\left[\beta_{0}, \beta_{1}, \ldots, \beta_{p}\right]^{\prime}
$$

in which the effect of each covariate is estimated as a fixed effect for any sample. As shown in Figure 8, the errors in the predicted lifetimes were much higher (that for sample $D 4$ exceeded 35\%). This is because covariate effects differ among subjects (drivers), operators (companies), and usage conditions. 
Table 5: Estimation results using equation 7

\begin{tabular}{l|cc}
\hline & Coef. & $p$-value \\
\hline Intercept & -15.453 & $<0.000$ \\
\hdashline Avg. GVW & 0.011 & 0.004 \\
Gradient & 2.866 & 0.091 \\
Avg. Speed & -0.017 & 0.035 \\
Avg. Accelr Pos. & 0.062 & $<0.000$ \\
Start Freq. & 0.119 & 0.352 \\
\hline
\end{tabular}

\begin{tabular}{ll}
\hline Residual Standard Err. & 0.227 \\
\hline Adjusted R-squared & 0.870 \\
\hline
\end{tabular}

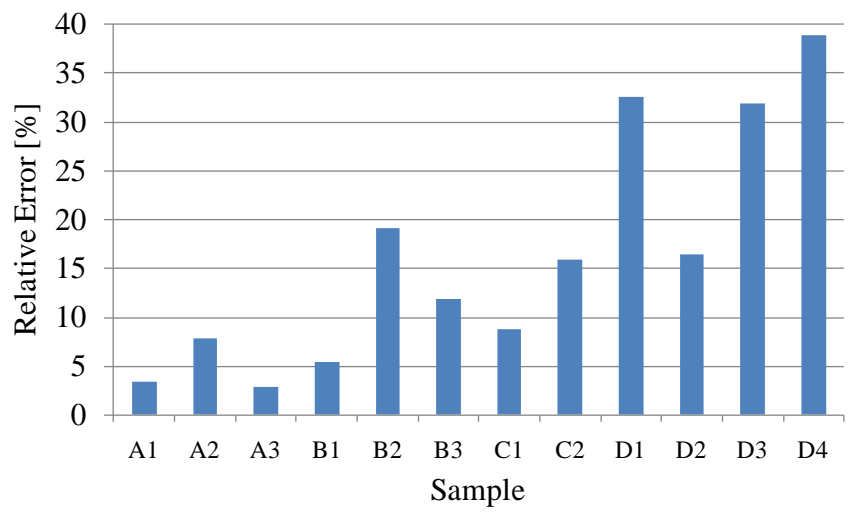

Figure 8: Relative error in predicted lifetime [\%] (Derived from equation 7)

As an idea to improve the accuracy of liner model, transformation of objective variables or introduction of the quadratic terms or other non-liner terms are considered from the results of performing the regression diagnostics. On the other hand, Kumazaki et al. (2014) ware modeled the variations between samples by applying the mixed-effects model. However, this study does not take into account of usefulness as the model to predict the lifetime for another sample. Therefore, this study applied stratified analysis as the simple approach to avoid the complexity due to the model extension.

\subsection{Stratified Analysis}

A simple and reasonable method to use when the covariate effects differ among certain groups is stratified analysis. We performed stratified analysis using two stratification factors: operator and usage.

\subsubsection{Stratification by Operator}

As mentioned above, the vehicles were operated by four companies. There were various differences among the operators, including the type of cargo normally carried and management style. These differences may be reflected in the degree of gear damage. In fact, the degree of damage (see Figure 3) tended to be similar among the vehicles of each operator. Therefore, we estimated a linear model by stratification into four groups (operator $A, B, C, D)$. The confidence intervals of the regression coefficients estimated for each operator's data for each covariate are shown in Figure 9. The covariates that did not overlap the confidence interval for each group were "Intercept," "Gradient," and "Avg. Accelr Pos." These results show that the covariate effects differed among operators. 


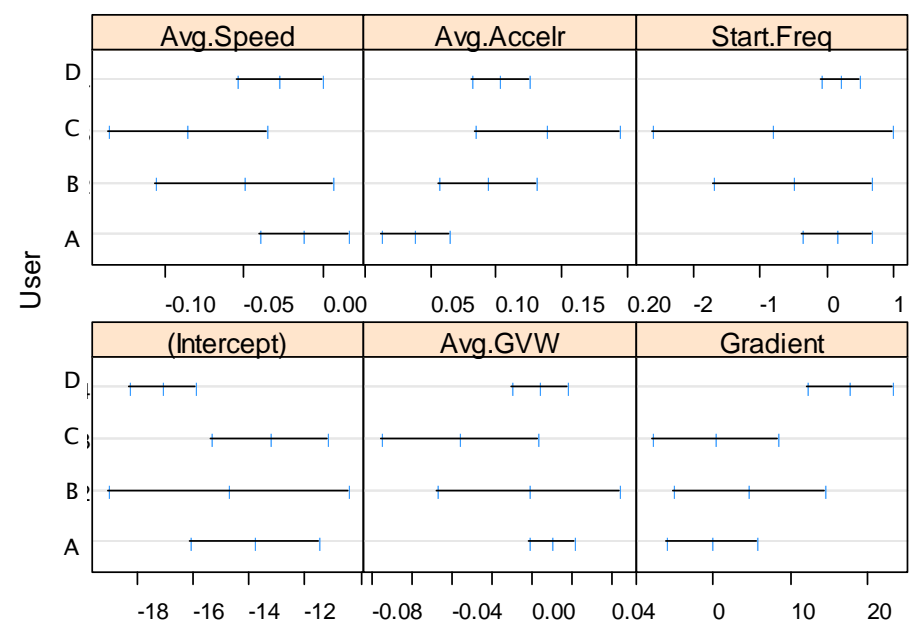

Figure 9: Confidence interval of regression coefficients by operator

\subsubsection{Stratification by Usage}

Stratification by operator is a simpler approach, but the model is based on only four operators and is thus not yet practical. Another possible approach is stratification based on differences in usage. Table 6 shows the results of principal component analysis on the covariates.

This analysis used ten variables that were chosen by eliminating the variables having high mutual correlation. The data used were the same as in the previous analysis, so each sample had 15 measurement points (except for one). Looking at the cumulative proportion, we see that the first to third principal components describes $80 \%$ of the data. The principal component scores and factor loadings are shown in Figure 10.

The chart on the left is for the first and second PCs, the one on the right is for the third and second PCs. In the figure, the plot points are the principal component scores for the data points. The representations for the 12 types of samples are shown in the figure legend. Focusing on the factor loadings (indicated by the arrows), we see that GVW, accelerator position angle, and engine RPM contribute to the first PC, so they reflect the load capacity. The 9th gear frequency, vehicle speed, shift frequency, and start frequency contribute to the second PC, so they reflect the characteristics of the route (e.g., highway driving vs. local road driving). Finally, the gradient contributes to the third PC, so it reflects the road gradient of the route. Looking at the principal component scores, we see that each sample's plot includes a variation of measurement point. The PCA results indicated differences in vehicle usage. Those of operator $A$ tended to carry a heavier load. Those of operator $D$, except for vehicle $D 2$, tended to travel flat local roads. Among the vehicles of operator $B$, only $B 1$ had slightly different characteristics.

Table 6: Results of principal component analysis (PC: principal component)

\begin{tabular}{clccccc}
\hline No & & PC 1 & PC 2 & PC 3 & PC 4 & PC 5 \\
\hline & Eigenvalue & 4.005 & 3.043 & 1.211 & 0.854 & 0.423 \\
& Proportion & 0.400 & 0.304 & 0.121 & 0.085 & 0.042 \\
& Cumulative Proportion & $\mathbf{0 . 4 0 0}$ & $\mathbf{0 . 7 0 5}$ & $\mathbf{0 . 8 2 6}$ & $\mathbf{0 . 9 1 1}$ & $\mathbf{0 . 9 5 4}$ \\
\hline 1 & Avg. GVW & 0.816 & -0.274 & -0.117 & -0.128 & 0.456 \\
2 & Gradient & 0.028 & 0.149 & 0.855 & 0.476 & 0.047 \\
3 & Avg. Speed & 0.607 & 0.765 & -0.073 & -0.052 & -0.115 \\
4 & Std. dev. Speed & 0.785 & -0.315 & 0.109 & -0.295 & -0.369 \\
5 & Avg. Engine RPM & 0.908 & 0.139 & -0.030 & 0.280 & -0.153 \\
6 & Std. dev. Engine RPM & 0.656 & -0.548 & -0.259 & 0.364 & -0.089 \\
7 & Avg. Accelr Pos. & 0.914 & 0.197 & 0.154 & -0.213 & 0.148 \\
8 & 9th Gear Freq & -0.223 & 0.867 & 0.132 & -0.336 & -0.051 \\
9 & Shift Freq. & 0.170 & -0.659 & 0.582 & -0.375 & -0.004 \\
10 & Start Freq. & -0.427 & -0.845 & -0.039 & -0.104 & -0.088 \\
\hline
\end{tabular}



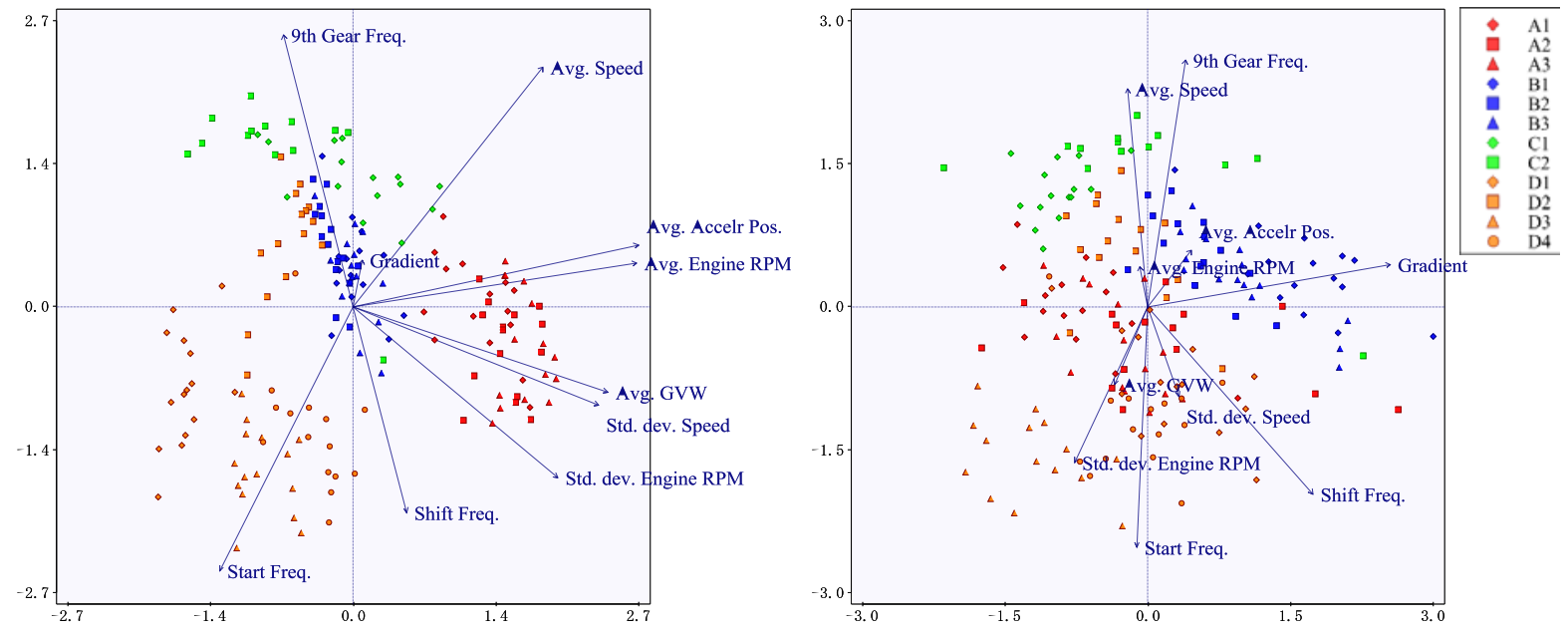

Figure 10: Principal component scores (plot points) and factor loadings (arrows)

We used next the Ward method and the principal component score to perform cluster analysis. The dendrogram shown in Figure 11 was obtained from hierarchical cluster analysis. The vertical axis represents the dissimilarity, and the horizontal axis represents each data point (labels omitted due to large number of data points). Neighboring data points were clustered into groups. The tree was cut at the level of the red dashed line, resulting in five clusters (I, II, III, IV, and V). Table 7 shows the results of cluster assignment. Then, the clusters assigned on the basis of highest frequency are shown in Table 8. Each sample was stratified on the basis of usage in accordance with the clustering results. The clusters are the stratification factors. The same as Figure 9, Figure 12 shows the confidence intervals of the regression coefficients estimated for each cluster. All the coefficient confidence intervals (except "Start Frequency") did not overlap for each group, indicating that the effect of the covariates differed among clusters.

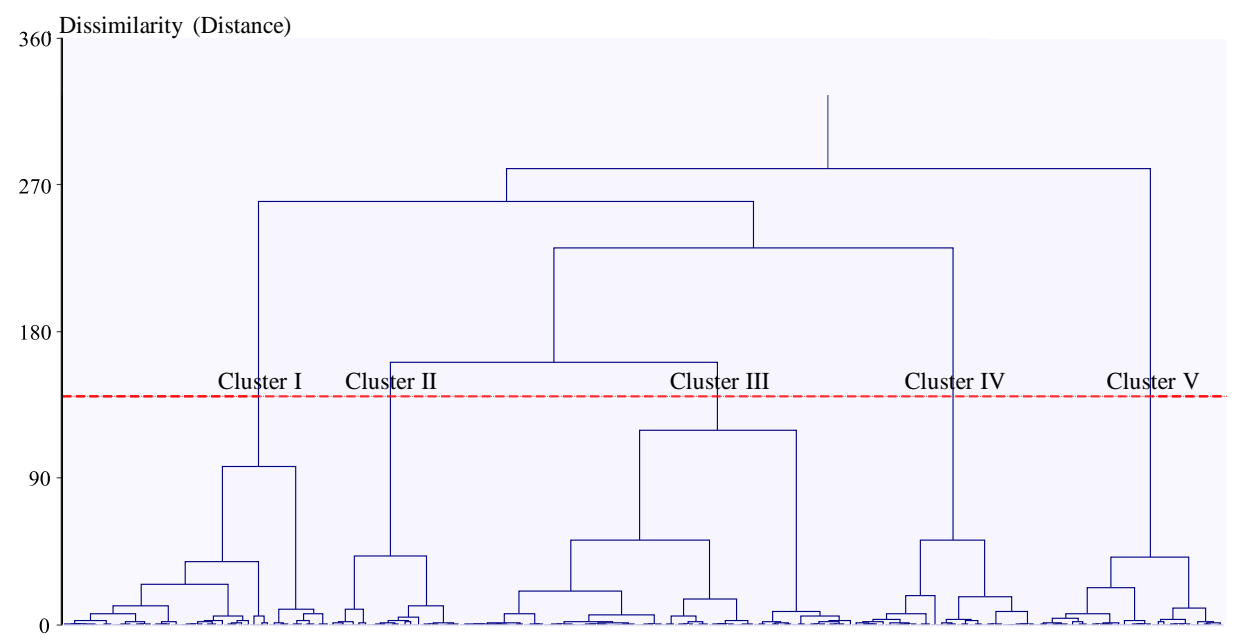

Figure 11: Results of cluster analysis (Dendrogram) 
Lifetime prediction using monitoring data, Kumazaki et al.

Table 7: Results of cluster assignment

\begin{tabular}{ccccccc}
\hline \multirow{2}{*}{ Sample } & Size & I & II & III & IV & V \\
\hline$A 1$ & 15 & 14 & 1 & 0 & 0 & 0 \\
$A 2$ & 15 & 10 & 5 & 0 & 0 & 0 \\
$A 3$ & 15 & 15 & 0 & 0 & 0 & 0 \\
$B 1$ & 15 & 0 & 13 & 1 & 1 & 0 \\
$B 2$ & 15 & 1 & 0 & 14 & 0 & 0 \\
$B 3$ & 15 & 0 & 0 & 15 & 0 & 0 \\
$C 1$ & 15 & 1 & 0 & 0 & 14 & 0 \\
$C 2$ & 13 & 0 & 1 & 0 & 12 & 0 \\
$D 1$ & 15 & 0 & 0 & 15 & 0 & 0 \\
$D 2$ & 15 & 0 & 0 & 15 & 0 & 0 \\
$D 3$ & 15 & 0 & 0 & 0 & 0 & 15 \\
$D 4$ & 15 & 0 & 0 & 0 & 1 & 14 \\
\hline
\end{tabular}

Table 8: Results of cluster assignment based on highest frequency

\begin{tabular}{ccccccccccccc}
\hline Sample & $A 1$ & $A 2$ & $A 3$ & $B 1$ & $B 2$ & $B 3$ & $C 1$ & $C 2$ & $D 1$ & $D 2$ & $D 3$ & $D 4$ \\
\hline Cluster & I & I & I & II & III & III & IV & IV & III & III & V & V \\
\hline
\end{tabular}

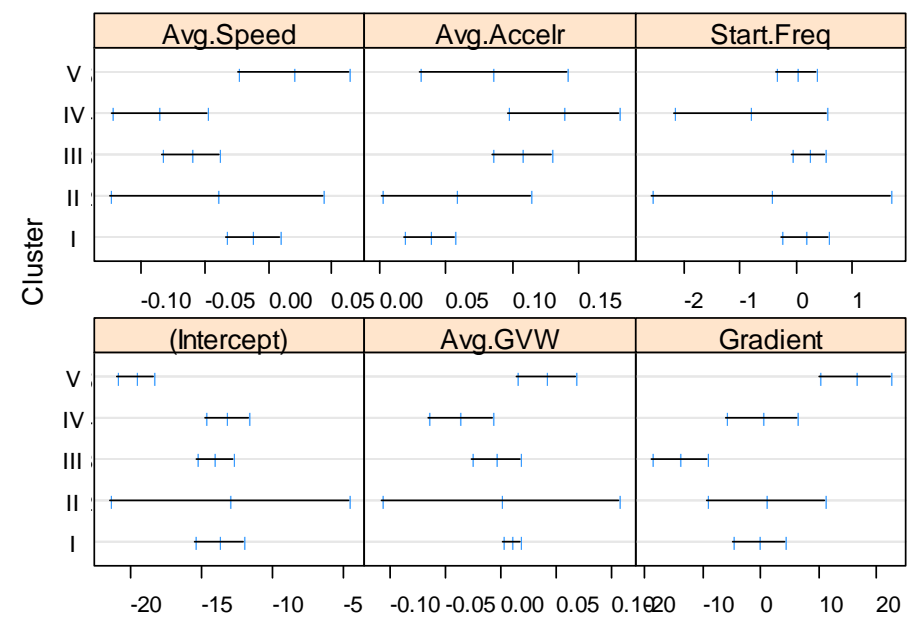

Figure 12: Confidence interval of regression coefficients of each cluster

\subsubsection{Prediction Results for Stratification}

The prediction results on the basis of the stratified analysis are shown in Table 9 and Figure 13 shows the maximum and average relative errors for 12 samples.

Table 9: Maximum and average relative errors in lifetime prediction

\begin{tabular}{c|c|c}
\hline & Max [\%] & Average [\%] \\
\hline Not stratified & 38.94 & 16.25 \\
\hline Stratified 1 & 28.67 & 8.44 \\
\hline Stratified 2 & 9.70 & 3.81 \\
\hline
\end{tabular}




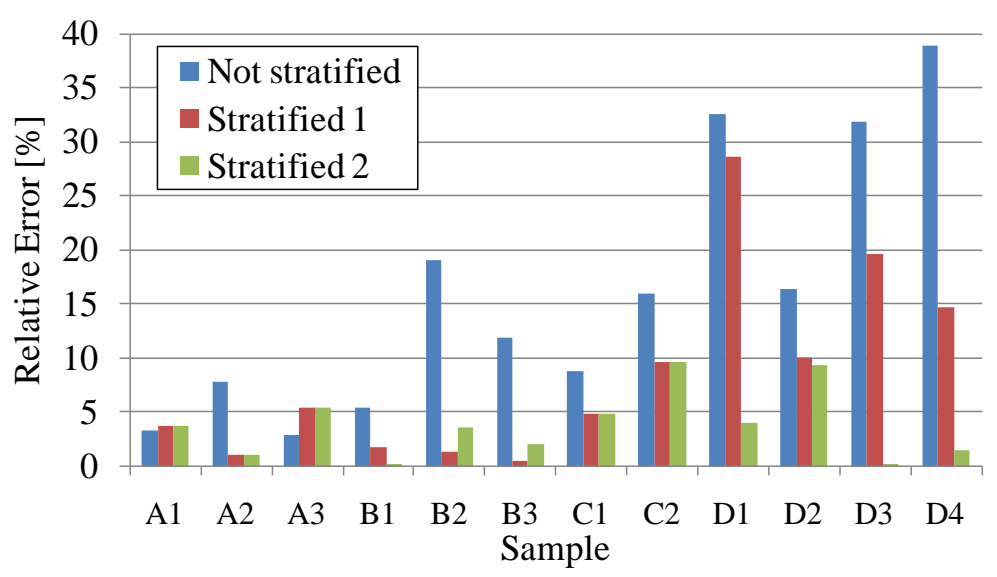

Figure 13: Relative error of prediction of lifetime [\%] (derived from stratified analysis)

In the figure, "Not stratified" means the results of using the generalization prediction procedure (Figure 8). "Stratified 1" means the results of stratification by operator (Section 4.2.1). It reduced the overall prediction error. "Stratified 2" means the results of stratification by usage (Section 4.2.2). It greatly reduced the overall prediction error. In particular, the most effective action is to stratify the vehicles of operator $D$ into two groups. In short, stratification based on usage improves prediction accuracy.

The proposed method performs clustering and principal component analysis by using covariates and predicts the degree of damage and lifetime from covariates. Application of this method, even to a new sample (even if only covariates), would enable groups to be determined and a prediction model to be fitted.

\section{Summary}

Our proposed method for predicting vehicle component lifetimes using an extensive amount of data collected during vehicle operation takes into account variations in usage conditions and models data as covariates. The generalized version is effective even for a new data sample. Our stratified analysis produced fairly accurate results and demonstrated that using online monitoring data is useful for predicting the lifetimes of vehicle components. The purpose of this system is to perform a reliable market evaluation for setting an appropriate target value at the design and development stage. To do this, the lifetime data will be required more and more. Therefore, more practical and effective operation of this system is required. Since the flow of application of this system could be shown, we will be able to develop to the actual business as the next step.

The validity of our sampling is problematic because only 12 vehicles were included in the sample. Sampling based on an experimental design is necessary. As mentioned in this study, market research using principal component analysis can take advantage about it.

\section{References:}

Everitt, B. S. (2005): An R and S-PLUS Companion to Multivariate Analysis, Springer.

Hong, Y. and W. Q. Meeker (2013): "Field-Failure Predictions Based on Failure-Time Data With Dynamic Covariate Information”, Technometrics, 55 (2), pp.135-149.

JSME S 002 (1994): Standard Method of Statistical Fatigue Testing (Revised Edition), The Japan Society of Mechanical Engineers (Japanese).

Kumazaki, C., M. Yokoyama, W. Yamamoto, and K. Suzuki (2012): "Optimal sampling plan and lifetime prediction based on online information," Shinraisei To Shisutemu Anzengaku (Proceeding of 16th Study of System Safety and Reliability), Graduate School of Information Systems, University of Electro-Communications, pp. 46-51 (Japanese).

Kumazaki, C., W. Yamamoto, and K. Suzuki (2014): "Lifetime prediction of vehicle components in considering variance of usage conditions based on online monitoring”, Proceeding of 44th Symposium on Reliability and Maintainability, Japan, pp.489-494 (Japanese).

Lee, J., F. Wu, W. Zhao, M. Ghaffari, L. Liao and D. Siegel (2014): "Prognostics and health management design for rotary machinery systems -Reviews, methodology and applications”, Mechanical Systems and Signal Processing, 42, pp.314-334. 
Lifetime prediction using monitoring data, Kumazaki et al.

Meeker, W. Q. and Y. Hong (2014): “Reliability Meets Big Data: Opportunities and Challenges,” Quality Engineering, 26 (1), pp. 102-116.

Mishra S., M. Pecht, T. Smith, I. McNee, and R. Harris (2002): "Remaining Life Prediction on Electronic Products using Life Consumption Monitoring Approach”, Proceedings of the European Microelectronics Packaging and Interconnection Symposium, Cracow, Poland, pp.136-142.

Pecht, M. and J. Gu (2009): "Physics-of-failure-based prognostics for electronic products", Transaction of the Institute of Measurement and Control, 31 (3-4), pp.309-322.

Suzuki, K. (2014): "Scheme of online monitoring and viewpoint of its effectiveness", in Proceeding of 3rd Memorial Lecture Series of Publication of "Reliability Handbook New Edition": Acquisition, Utilization and its Challenge of Reliability Big Data - The Scheme of Online Monitoring and its Future -, The 1st Forum in 2014 of Reliability Engineering Association of Japan, pp.15-43 (Japanese).

Suzuki, K. (2014): "Scheme of Reliability Engineering and Effectiveness of On-Line Monitoring Maintenance”, Proceedings of 6th Asia-Pacific International Symposium on Advanced Reliability and Maintenance Modeling, Sapporo.

Vichare N., P. Rodgers, V. Eveloy, and M. Pecht (2004): "In Situ Temperature Measurement of a Notebook Computer -A Case Study in Health and Usage Monitoring of Electronics”, IEEE Transactions on Device and Materials Reliability, 4(4), pp.658-663.

Wen Y., R. Wolski, and C. Krintz (2005): "Online Prediction of Battery Lifetime for Embedded and Mobile Device", in Power-Aware Computer Systems, Third International Workshop, PACS 2003, San Diego, CA, USA, 2003 Revised Papers, 3164, pp.57-72.

\section{Author's biographical notes}

Chiharu Kumazaki is a Ph.D. student at the Department of Informatics at the University of Electro-Communications, Japan. He is also an engineer of Hino Motors, Ltd.. His primary interest lies on the analysis of market data for the lifetime prediction of vehicle units.

Watalu Yamamoto is a lecturer at the Department of Informatics at the University of Electro-Communications, Japan. His research interests include industrial statistics for statistical quality control and reliability engineering and also applied statistics.

Kazuyuki Suzuki is a professor at the Department of Informatics at the University of Electro-Communications, Japan. He has been working on Reliability Engineering and Quality Management for over three decades. He has served as both the president of the Japanese Society for Quality Control (JSQC) in 2010 and 2011, and the president of Reliability Engineering Association of Japan (REAJ) in 2012 and 2013.

[DOI : 10.17929/tqs.1.52]

Received, May 20, 2014

Revised: November 28, 2014

Accepted: March 10, 2015 